\title{
Autonomic Nervous System Effects on the Atrioventricular Conduction Time - Heart Period Relationship During Physical Exercise and Passive Recovery
}

\author{
Grégory M Blain ${ }^{1,2}$, Guillaume P Ducrocq ${ }^{1}$, Mathilde Péraldi ${ }^{1}$, Olivier Meste ${ }^{2}$ \\ ${ }^{1}$ Université Côte d'Azur, LAMHESS, Nice, France \\ ${ }^{2}$ Université Côte d'Azur, CNRS, I3S, Sophia Antipolis, France
}

\begin{abstract}
During physical exercise and the following passive recovery, for a given heart period (i.e. RR interval), the atrioventricular conduction time (i.e. $P R$ interval) is known to be lengthened during recovery (i.e. hysteresis phenomenon). We aimed to determine non-invasively the role of the autonomic nervous system on this hysteresis.

20 healthy participants $(10$ males, 10 females $)$ performed, on separate days, two identical ramp exercise tests on a cycle ergometer to exhaustion $(233 \pm 56 \mathrm{~W})$. Following exercise, participants remained either seated on the bike or adopted a supine position for $30 \mathrm{~min}$. A 12-lead $E C G$ was monitored to determine RR and PR intervals. An ad-hoc PR estimator was used to limit potential $P$ wave detection error during intense exercise resulting from an overlapping $T$ wave.

A PR-RR hysteresis was observed in all participants, which area was $>3$ times more pronounced $(P<0.05)$ in the supine vs. the upright position. Our results showed that during recovery, the atrioventricular conduction time returned to a faster rate than the heart period toward resting values. This effect was greater in the supine position, when the vagal input to the heart is enhanced.

Our findings suggest that the PR-RR hysteresis might be explained by a greater vagal input at the atrioventricular compared to the sinus node during recovery.
\end{abstract}

\section{Introduction}

Electrocardiogram (ECG) measurements and analysis at rest and during physical exercise is a popular and useful tool for detecting physiological alteration of the heart function in health and disease. From the ECG signal, the sinus node depolarization rate can be estimated from the PP or RR intervals, whereas the atrioventricular conduction time of the action potentials can be estimated from the PR interval.

While RR interval time course during exercise and recovery has been intensively studied and used as a prognostic tool [1], [2], further insights into cardiac electrophysiology, such as atrial and ventricular conduction dynamics, can be obtained from the analysis of the PR interval. For example, during physical exercise, the reduction in PR interval with the increase in intensity is mainly linked to a withdrawal of the cardiac parasympathetic tone [3] and depict an improvement in conduction velocity through the myocardium. Because the detection of the P-wave occurrence is methodologically challenging during intense exercise and recovery [4], data about the dynamics of the atrioventricular conduction time are scarce. Nonetheless, findings from a population cohort study involving almost 2000 participants showed that estimating the PR interval from post-exercise recovery is clinically relevant because it is a more robust risk stratifier for cardiovascular death than the resting PR interval [5]. Thus, characterising the time course of the PR interval during exercise and the following recovery appears to be necessary to better determine the mechanisms modulating the atrioventricular conduction time.

Using a method we specifically developed to accurately estimate P-wave occurrence and PR interval during intense exercise and recovery, we previously showed that an hysteresis phenomenon exists in the PR vs. RR intervals relationship, when data from a maximal ramp exercise test and the following recovery are compared [4]. Moreover, the amplitude of this hysteresis was found to be larger in highly endurance trained compared to untrained participants. Because the improved cardiac recovery in trained endurance athlete is mainly linked to an improved vagal reengagement [6], these data suggest that the autonomic nervous system tonic input significantly contribute to the PR-RR intervals hysteresis. To noninvasively test this hypothesis, we modulated the orthostatic stress during post-exercise recovery and determined the influence of this modulation on the PR vs. $\mathrm{RR}$ intervals hysteresis.

\section{Methods}

\subsection{Participants}

Ten women (mean $\pm \mathrm{SD}$; age, $22 \pm 2$ years; maximal 
oxygen uptake $\left(\dot{\mathrm{V}} \mathrm{O}_{2 \max }\right), 34.2 \pm 4.6 \mathrm{ml} \cdot \mathrm{min}^{-1} \cdot \mathrm{kg}^{-1}$; peak power output, $3.1 \pm 0.3 \mathrm{~W} \cdot \mathrm{kg}^{-1}$ ) and ten men (age, $24 \pm 3$ years; $\dot{\mathrm{V}} \mathrm{O}_{2 \max }, 46.2 \pm 8.7 \mathrm{ml} . \mathrm{min}^{-1} . \mathrm{kg}^{-1}$; peak power output, $3.8 \pm 0.6 \mathrm{~W} . \mathrm{kg}^{-1}$ ) participated in the present study. All participants were students from a sports sciences faculty who trained in various sports from 2 to $8 \mathrm{~h}$ per week. All participants were non-smoker and were non-medicated. The study was approved by the local ethics committee and conducted according to the Declaration of Helsinki for human experimentation.

\subsection{Experimental design}

During one preliminary visit, anthropometric measurements were collected and subjects were familiarized with the exercise testing procedure. Participants performed a maximal ramp test $(60 \mathrm{~W}+1 \mathrm{~W}$ every four seconds until voluntary exhaustion) on a stationary cycle ergometer (Velotron, Elite Model; Racer Mate, Seattle, WA). To limit motion artifacts on the ECG signal from the upper limbs contractile activity and movements, the participant's forearms were positioned on a board placed in front of them at approximately the heart level, in a relaxed position. Participants were instructed to remain seated throughout exercise.

During the experimental visits, participants performed, on separate days, two identical maximal ramp cycling tests. During the first test, participants pedaled until voluntary exhaustion. $\dot{\mathrm{VO}}_{2 \max }$ and the peak power output that elicits $\dot{\mathrm{V}} \mathrm{O}_{2 \max }\left(\mathrm{P}_{\text {PEAK }}\right)$ were determined. During the second test, exercise was terminated when the power output equaled $\mathrm{P}_{\text {PEAK}}$. None of the participants failed to reached $\mathrm{P}_{\mathrm{PEAK}}$ during the second test. Prior the maximal cycling tests, cardiorespiratory data were collected at rest in the supine position and seated on the cycle ergometer during 15-min and 10-min, respectively. At exercise termination, during passive recovery, the cardiac autonomic nervous system activity was modulated by varying the level of the orthostatic stress. Cardiorespiratory data were randomly measured in the supine position (improved vagal return) or seated on the cycle ergometer (restrained vagal return) [7] during 30min. Experimental visits were performed at the same time of day. A randomized block design was used to account for potential order effects during the two experimental visits.

\subsection{Data collection and analysis}

At rest, during exercise and the following recovery, a twelve-lead ECG (Octal Bio-Amp, ML138; Ad Instrument, Bella-Vista, Australia) was recorded and the ECG signals were digitized at a sampling rate of $2 \mathrm{kHz}$. The ECG data was processed following chronological steps: we computed the PR and RR intervals, we normalised the $P R$ and $R R$ intervals and we finally quantified the hysteresis from the normalized intervals.

The RR interval estimation was achieved by using a threshold crossing technique or typical $\mathrm{R}$ wave detectors. Then the ECG was split in a set of RR segments. The PR interval was estimated by adapting a method that has been described in detail in a previous study [4]. Briefly, a reference $P$ wave was selected in the early part of exercise (i.e. at low exercise intensity) and was used as a template for a correlation based time delay estimation. In order to account for potential $\mathrm{T}$ and $\mathrm{P}$ waves overlapping, a reference $\mathrm{T}$ wave was also obtained from the ECG. It was optimally scaled and delayed, and finally removed from every RR segment. This optimization was performed together with the aforementioned time delay estimation.

During physical exercise, the increase in intensity reduces the ECG signal to noise ratio, mainly due to stronger muscles contractions and movements from the upper limb. To improve the signal to noise ratio and PR interval estimation accuracy, we averaged batches of 10 consecutive PR intervals. The same procedure was also applied to RR intervals.

Moreover, in order to compare PR and RR intervals from different participants, the following normalization procedure was applied. The series of $\operatorname{PR}(n)$ and $\operatorname{RR}(n)$ intervals were thus scaled and shifted to insure that the beginning and the end of the exercise equaled 1 and 0 , respectively (Fig. 1).

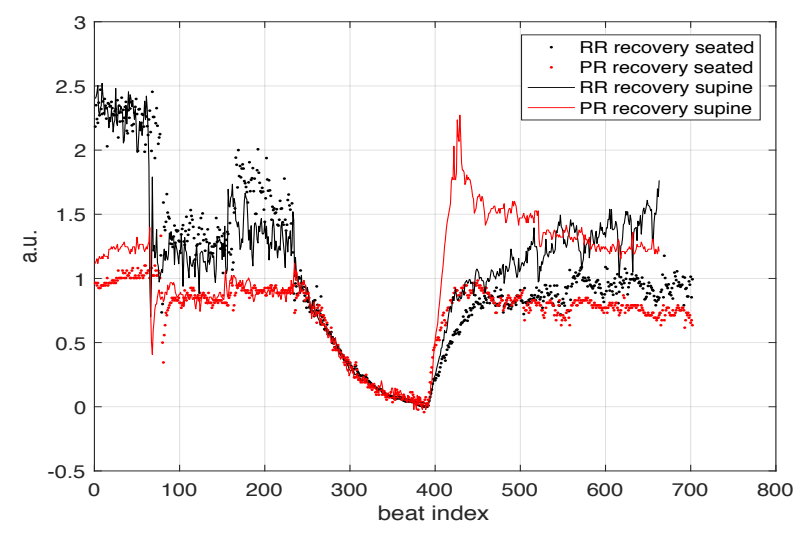

Figure 1. Normalized RR (black) and PR intervals (red) at rest, during exercise and post-exercise recovery in the supine (solid line) and seated (dots) positions. Note that 1) the RR and PR time courses during exercise were similar between-visits and 2) RR and PR intervals returned at a faster rate toward resting values in the supine position.

We then used a maximization approach on the normalized PR and RR intervals measure during exercise and recovery to quantify the PR-RR intervals hysteresis:

$$
\mathrm{T}_{\max }=\arg \max _{\mathrm{T}} \sum_{\mathrm{n}=\mathrm{n} 0}^{\mathrm{T}}(\operatorname{PR}(\mathrm{n})-\mathrm{RR}(\mathrm{n}))
$$


where $\mathrm{n} 0$ is the beat number corresponding to the beginning of the recovery.

Finally, the magnitude of the hysteresis $\mathrm{H}$ was determined using the following formula:

$$
H=\sum_{n=n 0}^{T_{\max }}(P R(n)-R R(n))
$$

\section{Results}

No significant between-visits difference $(p>0.62)$ was found in resting RR (supine: $1024 \pm 149 \mathrm{~ms}$; seated: $869 \pm$ $154 \mathrm{~ms}$ ) and PR (supine: $196 \pm 22 \mathrm{~ms}$; seated: $189 \pm 18$ $\mathrm{ms}$ ) as well as at peak exercise. Both RR and PR intervals progressively decreased during exercise and reached nadir values at peak power output $(327 \pm 13 \mathrm{~ms}$ and $149 \pm 16$ $m s$ for RR and PR, respectively). During passive recovery, $R R$ and PR intervals returned at a faster rate toward baseline values in the supine compared to the seated position (Fig. 1).

A significant hysteresis was observed in the PR intervals vs. RR intervals relationship, when the exercise and recovery data were computed together (Fig. 2 and 3). On average, the hysteresis magnitude $(\mathrm{H})$ was $>3$ times greater $(\mathrm{p}<0.02)$ in the supine $(52 \pm 49$ a.u. $)$ vs. the seated $(18 \pm 20$ a.u.) position. However, a great between-subjects variability was observed on the recovery position effect on the PR-RR hysteresis magnitude (Fig. 4). Over the 20 participants, $\mathrm{H}$ supine $-\mathrm{H}$ seated was $>0$ (i.e. the hysteresis magnitude was larger in the supine vs seated position) in 17 participants whereas it approximated 0 in the 3 remaining participants.

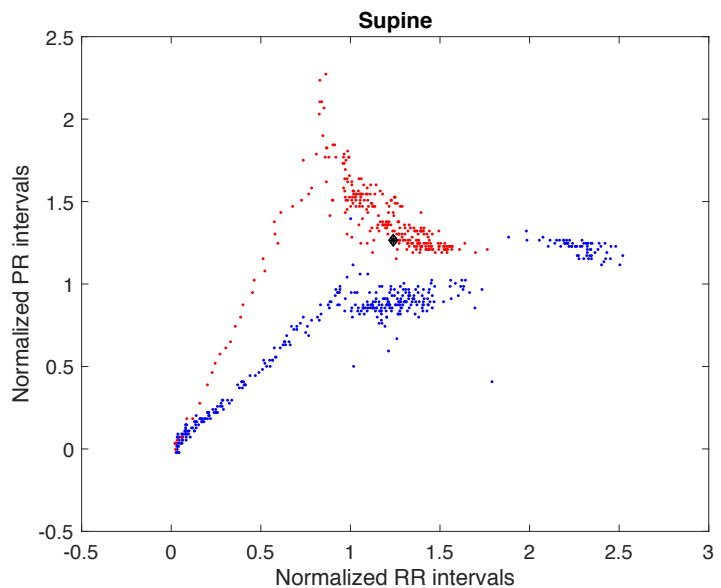

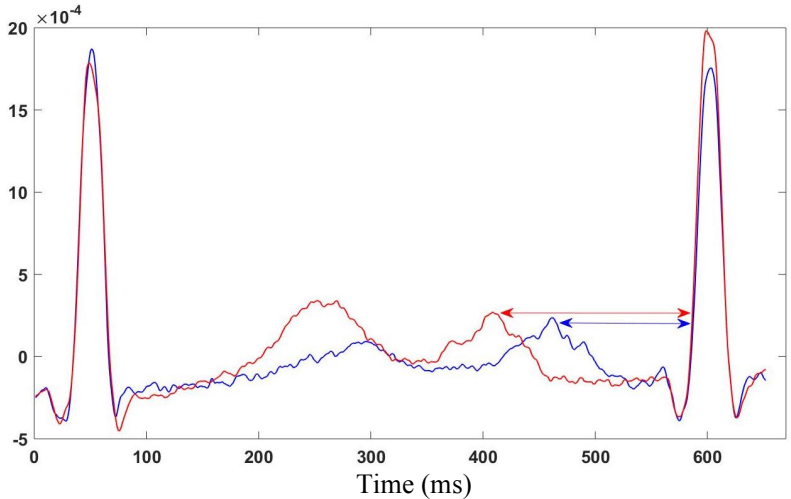

Figure 2. ECG signal for a given RR interval during exercise (blue line) and recovery (red line). Note that the PR interval was lengthened during recovery.

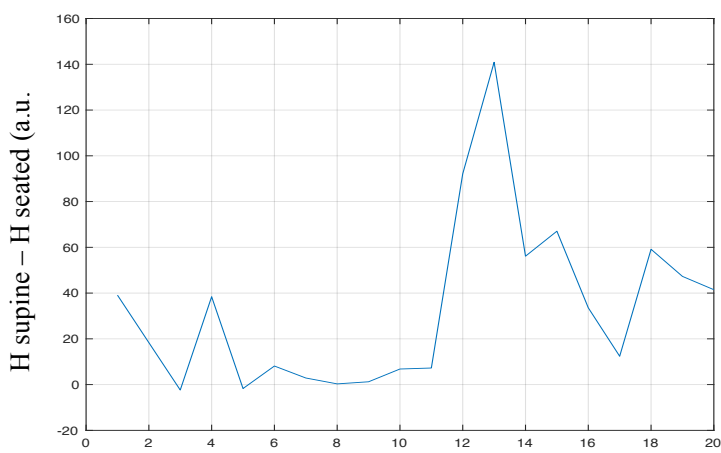

Participant number

Figure 4. Individual ditterence in the nysteresis magnitude in the supine vs. seated position (i.e. $\mathrm{H}$ supine $-\mathrm{H}$ seated).

\section{Discussion}

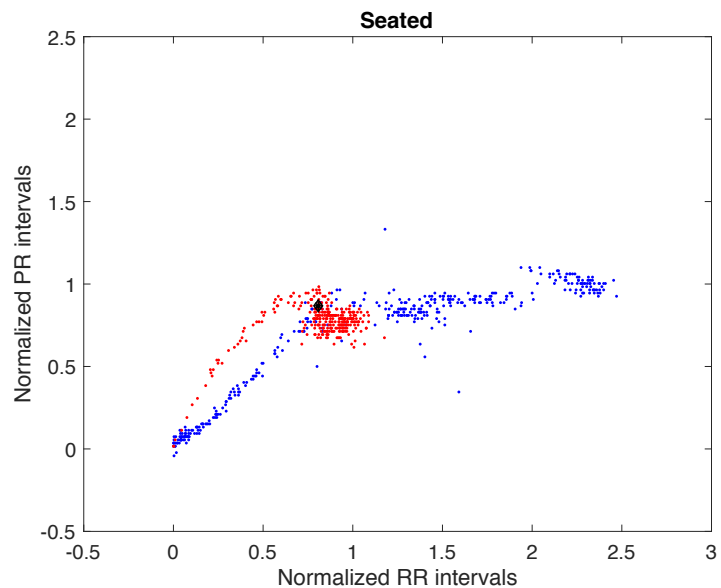

Figure 3. Normalized PR vs normalized RR intervals relationship during exercise (blue dots) and recovery (red dots). Recovery was performed in the supine (left graph) or in the seated (right graph) position. The black dot located approximately in the middle of each graph indicate the coordinates $P R\left(T_{\max }\right), R R\left(T_{\max }\right)$. Because the PR and RR intervals were normalized, the computed $\mathrm{H}$ is expressed in an arbitrary unit. Note the increased hysteresis in the supine position. 
We developed an experimental protocol to noninvasively test, in the healthy human, the influence of the autonomic nervous system on the relationship between the sinus and atrioventricular nodes activity (as estimated by the RR and PR intervals, respectively) during physical exercise and post-exercise recovery. Our findings showed that: 1) the PR vs. RR intervals relationship during a maximal ramp test exercise and the following passive recovery demonstrates an hysteresis pattern, and 2) the PR$\mathrm{RR}$ hysteresis magnitude is significantly more pronounced in the supine versus the seated position.

Considering that the difference in the ECG response to changes in the orthostatic stress (i.e. supine vs. seated) is mediated by changes in the autonomic nervous system tonic input [7], we interpret our findings of a greater hysteresis magnitude in the supine position as indirect evidence of a higher parasympathetic re-engagement and/or a larger sympathetic withdrawal at the atrioventricular compared to the sinus node during recovery. This interpretation is supported by previous neuroanatomical and electrophysiological findings which demonstrated that the autonomic nervous system neural circuitry which innervates the atrioventricular and the sinus nodes is specific and independent [8], [9]. Moreover, it is also consistent with previous findings showing, using pharmacological blockade in patients with implanted dualchamber devices, that the parasympathetic effect on the atrioventricular node was poorly correlated to the effect occurring at the sinus node during exercise and recovery.

\section{Implications and conclusion}

The study of the PR-RR intervals relationship during exercise and passive recovery revealed an hysteresis pattern (i.e. for a given RR interval during exercise and recovery, the PR interval was lengthened during recovery). This PR-RR hysteresis was strengthened when the participants performed the recovery in the supine compared to the seated position. Our results thus suggest that the PR-RR hysteresis appears to be mediated, at least in part, by an increase in the vagal tone and/or a reduction in the sympathetic tonic input, which are characteristics of the supine position.

On a clinical perspective, the lengthening at a fast rate of the atrioventricular conduction time during recovery in the supine position might indicate that the filtering role played by the atrioventricular node is reinforced following physical exercise, in response to rapid changes in the autonomic nervous system activity. This might in turn limit the risk of ventricular fibrillation and sudden cardiac death [10] that is known to be significantly increased compared to rest during physical exercise and recovery [11]. Further work is necessary to test this hypothesis and determine the clinical relevance of the PR-RR hysteresis as a diagnostic / prognostic tool in cardiology and exercise testing.

\section{Acknowledgments}

This work has been supported by the French government, through the UCAJEDI Investments in the Future project managed by the National Research Agency (ANR) with the reference number ANR-15-IDEX-01.

\section{References}

[1] S. Paolillo et al., " Heart rate during exercise: mechanisms, behavior, and therapeutic and prognostic implications in heart failure patients with reduced ejection fraction », Heart Fail. Rev., vol. 23, nº 4, p. 537- 545, juill. 2018.

[2] C. R. Cole, E. H. Blackstone, F. J. Pashkow, C. E. Snader, et M. S. Lauer, « Heart-rate recovery immediately after exercise as a predictor of mortality », in N Engl J Med, vol. 341, 1999, p. 1351- 7.

[3] J.-H. Atterhög et E. Loogna, « P-R interval in relation to heart rate during exercise and the influence of posture and autonomic tone », J. Electrocardiol., vol. 10, n ${ }^{\circ}$, p. 331- 336, janv. 1977.

[4] A. Cabasson, O. Meste, G. Blain, et S. Bermon, " Quantifying the PR interval pattern during dynamic exercise and recovery ", IEEE Trans. Biomed. Eng., vol. 56, $\mathrm{n}^{\mathrm{o}}$ 11, p. 2675- 2683, nov. 2009.

[5] T. Nieminen et al., « Atrioventricular conduction and cardiovascular mortality: Assessment of recovery PR interval is superior to pre-exercise measurement ", Heart Rhythm, vol. 7, $\mathrm{n}^{\circ}$ 6, p. 796- 801, juin 2010.

[6] J. Borresen et M. I. Lambert, « Autonomic Control of Heart Rate during and after Exercise: Measurements and Implications for Monitoring Training Status », Sports Med., vol. 38, n ${ }^{\circ} 8$, p. 633- 646, 2008.

[7] N. Montano, T. G. Ruscone, A. Porta, F. Lombardi, M. Pagani, et A. Malliani, « Power spectrum analysis of heart rate variability to assess the changes in sympathovagal balance during graded orthostatic tilt. ", Circulation, vol. 90, $\mathrm{n}^{\mathrm{o}} 4, \mathrm{p}$. 1826- 1831, oct. 1994.

[8] H. Inoue et D. P. Zipes, "Changes in atrial and ventricular refractoriness and in atrioventricular nodal conduction produced by combinations of vagal and sympathetic stimulation that result in a constant spontaneous sinus cycle length », Circ Res, vol. 60, nº 6, p. 942- 51, juin 1987.

[9] A. D'Avila, M. Scanavacca, E. Sosa, J. N. Ruskin, et V. Y. Reddy, "Pericardial anatomy for the interventional electrophysiologist », J Cardiovasc Electrophysiol, vol. 14, $\mathrm{n}^{\circ} 4$, p. 422- 30, avr. 2003.

[10] F. L. Meijler, I. van der Twell, J. N. Herbschleb, R. N. W. Hauer, et E. O. Robles de Medina, « Role of atrial fibrillation and atrioventricular conduction (including Wolff-ParkinsonWhite syndrome) in sudden death », J. Am. Coll. Cardiol., vol. 5, $\mathrm{n}^{\mathrm{o}}$ 6, p. 17B-22B, juin 1985.

[11] C. M. Albert, M. A. Mittleman, C. U. Chae, I. M. Lee, C. H. Hennekens, et J. E. Manson, « Triggering of sudden death from cardiac causes by vigorous exertion », N. Engl. J. Med., vol. $343, n^{\circ} 19$, p. 1355- 1361, nov. 2000.

Address for correspondence: Grégory Blain.

LAMHESS - Faculté des Sciences du Sport, 261 boulevard du Mercantour, 06205 Nice, France gregory.blain@univcotedazur.fr 